BRIEF REPORT

\title{
Is Swedish snus associated with smoking initiation or smoking cessation?
}

\author{
H Furberg, C M Bulik, C Lerman, P Lichtenstein, N L Pedersen, P F Sullivan
}

Tobacco Control 2005;14:422-424. doi: 10.1136/tc.2005.012476

Nicotine replacement therapies (NRT) are an effective treatment for tobacco dependence, yet most smokers do not quit or remain abstinent. We investigated whether Swedish snus (snuff) use was associated with smoking cessation among males participating in a large population based twin study in Sweden. Snus use was associated with smoking cessation but not initiation. Given that snus delivers comparable nicotine concentrations but carries lesser cancer risk than cigarettes, snus may be a widely used, non-medical form of NRT. Evaluation of the efficacy of snus for smoking cessation should be evaluated in randomised clinical trials.

$\mathrm{T}$ bacco use is the second major cause of death worldwide. Nicotine replacement therapy (NRT) can double smoking cessation rates, but most smokers receiving treatment do not achieve long term abstinence. ${ }^{1}$ Additional NRT is needed.

The prevalence of cigarette smoking is notably low in Sweden while the use of snus is increasing. Swedish snus is a moist smokeless tobacco product that contains lower concentrations of cancer-causing tobacco-specific nitrosamines than found in other smokeless tobacco products and cigarettes. ${ }^{2}$ While snus delivers similar concentrations of nicotine, it carries substantially lower risks of cancer than cigarettes. $^{3-8}$ Male smokers in Sweden appear to be using snus as a form of NRT, ${ }^{9}$ despite a lack of data from randomised clinical trials to support its use as a smoking cessation treatment. ${ }^{10}$

We investigated whether lifetime snus use was associated with smoking initiation or cessation to gain insight into its potential role as NRT. It is critical to examine snus' potential dual effects on smoking, given the fear that advocating the use of snus might increase smoking, thereby mitigating its utility as NRT. ${ }^{11-13}$

\section{PARTICIPANTS, METHODS AND RESULTS}

Data from the screening across lifespan twin study (SALT) from the population based Swedish Twin Registry were used. ${ }^{14}$ The study protocol was reviewed and approved by the ethical committee of the Karolinska Institute, the Swedish Data Inspection Board, and the Institutional Review Board at the University of North Carolina at Chapel Hill.

All subjects provided written informed consent. Briefly, SALT contains detailed data on tobacco use (type, amount, age at first use, patterns of use, and Fagerstrom test for nicotine dependence (FTND)) from telephone interviews completed by 31425 twins born in Sweden before 1959. Participants who currently or formerly "smoke(d) at parties", "smoke(d) now and then", or "smoke(d) regularly" were considered "ever smokers". Participants who "only tried cigarettes" or "never smoked" were considered "never smokers". We classified participants as "ever snus user" if they currently or formerly used snus "now and then" or "regularly"; otherwise they were classified as "never snus user". The questionnaire did not specifically describe what was meant by "regular" or "now and then" tobacco use, rather it was up to the participant to interpret and select the type of tobacco user they considered themselves to be. Since the lifetime prevalence of any snus use was only $2.5 \%$ among females, we restricted our analyses to males $(n=14932)$.

To investigate whether snus use was associated with smoking initiation, we compared men who used snus before they started smoking to men who never used snus in relation to any lifetime smoking (ever versus never "regular" or "now and then" cigarette smokers). To address whether snus use was associated with smoking cessation, we compared men who used snus after they began smoking to men who never used snus in relation to smoking status at the time of interview (former versus current "regular" or "now and then" smokers).

Odds ratios (OR) and 95\% confidence intervals (CI) were estimated with age adjusted logistic regression models and generalised estimating equations to account for clustering of twin pairs. Stratified analyses were performed to examine whether the associations for smoking cessation remained in subgroups of smokers (heavy versus light smokers; high versus low FTND scores).

Table 1 presents the distributions of tobacco use. Of the 14932 males that participated in SALT, 14424 (96.6\%) had tobacco use data and ages at initiation. Of these men, 9151 $(63.5 \%)$ reported smoking during their lifetime. Of the smokers, $7880(86.1 \%)$ reported that they smoke(d) "regularly", 669 (7.3\%) smoke(d) "now and then", and 602 $(6.6 \%)$ "smoke(d) at parties". The prevalence of current smoking status was highest for "now and then" smokers $(39.2 \%)$ as compared with "regular" smokers (34.0\%) and "party" smokers (23.1\%). The prevalence of any lifetime snus use in SALT was $28.5 \%(\mathrm{n}=4119)$, the majority of whom used snus regularly $(\mathrm{n}=3704,(89.9 \%))$.

"Regular" and "now and then" snus use were inversely associated with smoking initiation (table 2 ). Only $4.1 \%$ of men who ever smoked used snus "regularly" before they started smoking, while $18.5 \%$ of non-smokers had used snus "regularly". The odds ratio (OR) for "regular" snus use and ever smoking status was 0.2 ( $95 \%$ confidence interval (CI) 0.2 to 0.3 ). Despite smaller sample sizes, a similar pattern was observed for men who reported they used snus "now and then". Only $0.5 \%$ of men who ever smoked used snus "now and then" before they started smoking, while $1.1 \%$ of never smokers reported that they used snus "now and then". "Now and then" snus use was also inversely associated with ever smoking status (OR $0.5,95 \%$ CI 0.3 to 0.7 ), suggesting that men who used snus "regularly" or "now and then" before they began smoking were less likely to ever smoke.

Abbreviations: FTND, Fagerstrom test for nicotine dependence; NRT, nicotine replacement therapy; SALT, screening across lifespan twin study 
Table 1 Distributions of cigarette smoking and snus use among males in the Swedish Twin Registry

\begin{tabular}{|c|c|c|c|c|c|c|c|}
\hline & \multicolumn{6}{|c|}{ Ever smoker ( $n=9151,63.5 \%)$} & \multirow{3}{*}{$\begin{array}{l}\text { Never smoker } \\
(n=5273,36.5 \%)\end{array}$} \\
\hline & \multicolumn{2}{|l|}{$\begin{array}{l}\text { Ever regular smoker } \\
\text { ( } \mathrm{n}=7880,86.1 \%)\end{array}$} & \multicolumn{2}{|c|}{$\begin{array}{l}\text { Ever now and then smoker } \\
(\mathrm{n}=669,7.3 \%)\end{array}$} & \multicolumn{2}{|c|}{$\begin{array}{l}\text { Ever party smoker* } \\
(n=602,6.6 \%)\end{array}$} & \\
\hline & $\begin{array}{l}\text { Current regular } \\
\text { smoker } \\
(\mathrm{n}=2683,34.0 \%)\end{array}$ & $\begin{array}{l}\text { Former regular } \\
\text { smoker } \\
\text { (n=5197, 66.0\%) }\end{array}$ & $\begin{array}{l}\text { Current now and } \\
\text { then smoker } \\
\text { ( } \mathrm{n}=262,39.2 \%)\end{array}$ & $\begin{array}{l}\text { Former now and } \\
\text { then smoker } \\
\text { ( } \mathrm{n}=407,60.8 \% \text { ) }\end{array}$ & $\begin{array}{l}\text { Current party } \\
\text { smoker } \\
\text { ( } n=139,23.1 \% \text { ) }\end{array}$ & $\begin{array}{l}\text { Former party } \\
\text { smoker: } \\
\text { (n=463, 76.9\%) }\end{array}$ & \\
\hline \multicolumn{8}{|l|}{ Regular snus use ( $n=3704,25.6 \%)$} \\
\hline Began using snus before cigarettes & 61 & 147 & 16 & 38 & . & . & NA \\
\hline Began using snus after cigarettes & 324 & 1701 & 57 & 70 & . & . & NA \\
\hline Only used snus, no cigarettes & NA & NA & NA & NA & NA & NA & 976 \\
\hline Began using both at same time & 47 & 230 & 13 & 24 & . & . & NA \\
\hline \multicolumn{8}{|l|}{$\begin{array}{l}\text { Now and then snus use ( } n=415 \text {, } \\
2.9 \%)\end{array}$} \\
\hline Began using snus before cigarettes & 11 & 10 & 2 & 6 & . & . & NA \\
\hline Began using snus after cigarettes & 100 & 137 & 13 & 20 & . & . & NA \\
\hline Only used snus, no cigarettes & NA & NA & NA & NA & NA & NA & 60 \\
\hline Began using both at same time & 20 & 22 & 3 & 11 & . & . & NA \\
\hline Never used snus ( $n=10305,71.5 \%$ ) & 2120 & 2950 & 158 & 238 & 139 & 463 & 4237 \\
\hline
\end{tabular}

Table 2 Age adjusted odds ratios (OR) (95\% confidence intervals (CI)) for "regular" and "now and then" snus use in relation to any lifetime cigarette smoking among males in the Swedish Twin Registry

\begin{tabular}{llllll}
\hline & \multicolumn{1}{l}{ Ever smoker* } & Never smoker & OR (95\%Cl) \\
\hline Regular snus use & $262 \dagger$ & $4.1 \%$ & 976 & $18.5 \%$ & $0.2(0.2$ to 0.3$)$ \\
Now and then snus use & $29 \dagger$ & $0.5 \%$ & 60 & $1.1 \%$ & 0.5 (0.3 to 0.7) \\
Never used snus & 5466 & $95.4 \%$ & 4237 & $80.4 \%$ & 1.0 \\
\hline *'Ever smoker" includes men who reported smoking "regularly" and "now and then". Men who smoked "at \\
parties" were excluded since they did not contribute ages at onset of tobacco use. \\
†Men who began using snus before cigarettes.
\end{tabular}

"Regular" snus use was associated with smoking cessation among "regular" and "now and then" smokers (table 3). The proportion of former smokers who used snus "regularly" $(34.6 \%)$ was higher than the proportion of current smokers who used snus regularly (13.7\%). The OR for "regular" snus use and former smoking status was 3.7 (95\% CI 3.3 to 4.2 ), indicating that men who used snus "regularly" were over three times more likely to be former smokers than current smokers. No association was observed between "now and then" snus use and smoking cessation (OR 1.1, 95\% CI 0.9 to 1.4). Thus, it appears that only "regular" snus use has an impact on smoking cessation.

\section{DISCUSSION}

Consistent with recent studies, ${ }^{2}{ }^{15-19}$ we observed that snus use was associated with smoking cessation, not initiation. Our results support the idea that snus is a type of naturalistic and non-medical NRT that smokers in Sweden may be using to enhance smoking cessation efforts. ${ }^{9}$ We acknowledge the cross sectional nature of our data and assert that this correlation is not necessarily causal. Taken together with the information presented in a recent debate over the potential of snus as a smoking cessation aide, ${ }^{2}$ we suggest that randomised clinical trials are needed to investigate the utility and risks of snus as NRT under controlled conditions.

We are aware that advocating the use of one addictive tobacco product to diminish the harm from another is a controversial issue, particularly as data supporting the use of snus as NRT could enhance the market of the tobacco industry. Clearly, eliminating all forms of tobacco use would have the most beneficial impacts on world health; however, many smokers are unable to achieve lasting smoking cessation. From a harm reduction perspective, should snus be shown to be as effective as or superior in efficacy to existing NRTs without having adverse health consequences, it may represent a more acceptable means by which to reduce tobacco related health burden.

\section{ACKNOWLEDGEMENTS}

This work was supported by CA-085839 to PFS. The funding source had no involvement in study design, collection, analysis, interpretation of the data, and was not involved in the decision to submit this paper for publication.

Table 3 Age adjusted OR (95\% Cl) for smoking cessation (former versus current smokers) by snus use among males in the Swedish Twin Registry

\begin{tabular}{llllll}
\hline & \multicolumn{2}{l}{ Former smoker } & \multicolumn{2}{l}{ Current smoker } & OR (95\% Cl) \\
\hline Regular snus use & $1771 \dagger$ & $34.6 \%$ & $381 \dagger$ & $13.7 \%$ & 3.7 (3.3 to 4.2) \\
Now and then snus use & $157 \dagger$ & $3.1 \%$ & $113 \dagger$ & $4.1 \%$ & 1.1 (0.9 to 1.4) \\
Never used snus & 3188 & $62.3 \%$ & 2278 & $82.1 \%$ & 1.0 \\
\hline
\end{tabular}

*"Former smokers" and "current smokers" includes men who reported smoking "regularly" and "now and then" Men who smoked "at parties" were excluded since they did not contribute ages at onset of tobacco use. †Men who began using snus after cigarettes. 


\section{What this paper adds}

This brief report presents findings that Swedish snus (oral snuff) is being used as a naturalistic form of nicotine replacement therapy. We suggest that randomised clinical trials be conducted to assess the efficacy of snus as a smoking cessation aide and to evaluate whether any adverse health consequences result.

\section{CONTRIBUTORS}

$\mathrm{H}$ Furberg conducted the data analysis and wrote the manuscript. C Bulik provided critical revision for the manuscript for important intellectual content. C Lerman assisted in conception and analytic strategy of research question and contributed to manuscript revisions. P Lichtenstein and $\mathrm{N}$ Pedersen assisted in conception and analytic strategy of research question, contributed to manuscript revisions and provided the data from the Swedish Twin Registry. $\mathrm{P}$ Sullivan funded the analysis, supervised the data analysis and contributed to manuscript revisions. All authors reviewed and approved the final version of the manuscript.

\section{Authors' affiliations}

H Furberg, P F Sullivan*, Department of Genetics, University of North Carolina at Chapel Hill, Chapel Hill, North Carolina, USA

C M Bulik, Department of Psychiatry, University of North Carolina at Chapel Hill

C Lerman, Department of Psychiatry, Transdisciplinary Tobacco Use Research Center, University of Pennsylvania, Pennsylvania, USA P Lichtenstein, N L Pedersent, Department of Medical Epidemiology and Biostatistics, Karolinska Institute, Stockholm, Sweden

*Also Department of Medical Epidemiology and Biostatistics, Karolinska Institute, Stockholm, Sweden; †Also Department of Psychology, University of Southern California, California, USA

Competing interest: None of the authors has a conflict of interest that could inappropriately bias the work. In the late 1970s the Council for Tobacco Research funded collection of data used by N Pedersen in her dissertation. P Sullivan had full access to all the data in the study and had final responsibility for the decision to submit for publication.

Correspondence to: Dr Patrick F Sullivan, Department of Genetics, CB\#7264, 4109D Neurosciences Research Building, University of North Carolina, Chapel Hill, NC, 27599-7264, USA; pfsulliv@med.unc.edu
Received 12 May 2005

Accepted 10 August 2005

\section{REFERENCES}

1 Silagy C, Lancaster T, Stead L, et al. Nicotine replacement therapy for smoking cessation. Cochrane Database Syst Rev 2004(3):CD000146.

2 Foulds J, Ramstrom L, Burke M, et al. Effect of smokeless tobacco (snus) on smoking and public health in Sweden. Tobacco Control 2003;12:349-59.

3 Lewin $\mathrm{F}$, Norell SE, Johansson $\mathrm{H}$, et al. Smoking tobacco, oral snuff, and alcohol in the etiology of squamous cell carcinoma of the head and neck: a population-based case-referent study in Sweden. Cancer 1998;82:1367-75.

4 Schildt EB, Eriksson M, Hardell L, et al. Oral snuff, smoking habits and alcohol consumption in relation to oral cancer in a Swedish case-control study. Int J Cancer 1998;77:341-6.

5 Ye W, Ekstrom AM, Hansson LE, et al. Tobacco, alcohol and the risk of gastric cancer by sub-site and histologic type. Int J Cancer 1999;83:223-9.

6 Lagergren J, Bergstrom R, Lindgren A, et al. The role of tobacco, snuff and alcohol use in the aetiology of cancer of the oesophagus and gastric cardia. Int J Cancer 2000;85:340-6.

7 Accortt NA, Waterbor JW, Beall C, et al. Chronic disease mortality in a cohort of smokeless tobacco users. Am J Epidemiol 2002;156:730-7.

8 Rodu B, Jansson C. Smokeless tobacco and oral cancer: a review of the risks and determinants. Crit Rev Oral Biol Med 2004;15:252-63.

9 Ramstrom L. Patterns of use: a gate leading to smoking or a way out? Nicotine Tob Res 2003;5:268.

10 Fagerstrom KO, Schildt EB. Should the European Union lift the ban on snus? Evidence from the Swedish experience. Addiction 2003;98:1191-5.

11 Tomar SL. Smokeless tobacco use is a significant predictor of smoking when appropriately modeled. Nicotine Tob Res 2003;5:571-3.

12 Stratton K, Shetty P, Wallace R, et al. Clearing the smoke: the science base for tobacco harm reduction-executive summary. Tobacco Control 2001;10:189-95.

13 Haddock CK, Weg MV, DeBon M, et al. Evidence that smokeless tobacco use is a gateway for smoking initiation in young adult males. Prev Med $2001 ; 32: 262-7$.

14 Lichtenstein P, De Faire U, Floderus B, et al. The Swedish Twin Registry: a unique resource for clinical, epidemiological and genetic studies. J Intern Med 2002;252:184-205.

15 Gilljam H, Galanti MR. Role of snus (oral moist snuff) in smoking cessation and smoking reduction in Sweden. Addiction 2003;98:1183-9.

16 Ramstrom L. Snus: part of the problem or part of the solution? Addiction 2003;98: 1198-9, discussion 1204-7.

17 Rodu B, Stegmayr B, Nasic S, et al. Evolving patterns of tobacco use in northern Sweden. J Intern Med 2003;253:660-5.

18 Kozlowski LT, O'Connor RJ, Edwards BQ, et al. Most smokeless tobacco use is not a causal gateway to cigarettes: using order of product use to evaluate causation in a national US sample. Addiction 2003;98:1077-85

19 O'Connor RJ, Kozlowski LT, Flaherty BP, et al. Most smokeless tobacco use does not cause cigarette smoking: results from the 2000 National Household Survey on Drug Abuse. Addict Behav 2005;30:325-36. 